\title{
Comparing Usage of a Large High-Resolution Display to Single or Dual Desktop Displays for Daily Work
}

\author{
Xiaojun Bi, Ravin Balakrishnan \\ Department of Computer Science \\ University of Toronto \\ \{xiaojun, ravin\}@dgp.toronto.edu
}

\begin{abstract}
With the ever increasing amount of digital information, users desire more screen real estate to process their daily computing work, and might well benefit from using a wallsize large high-resolution display instead of a desktop one. Unfortunately, we know very little about users' behaviors when using such a display for daily computing. We present a week-long study that investigates large display use in a personal desktop computing context by comparing it with single and dual desktop monitor use. Results show users' unanimous preference for using a large display: it facilitates multi-window and rich information tasks, enhances users' awareness of peripheral applications, and offers a more "immersive" experience. Further, the data reveals distinct usage patterns in partitioning screen real estate and managing windows on a large display. Detailed analysis of these results provides insights into designing interaction techniques and window management systems more suited to a large display.
\end{abstract}

\section{Author Keywords}

Large display, Personal desktop work

\section{ACM Classification Keywords}

H5.m. Information interfaces and presentation (e.g., HCI): Miscellaneous.

\section{INTRODUCTION}

As users increasingly juggle multiple applications processing different types of information, they tend to benefit from more screen real estate for their desktop work. Over the past decade, the default size of a desktop monitor has increased from $15^{\prime \prime}$ to 21 ", and users are also beginning to rely more on multiple monitors [10]. Indeed, recent research has shown the benefits of using larger displays for personal desktop work $[8,9]$.

The rapid advancement of display technology makes ever larger high-resolution displays increasingly available and affordable. Currently, one of the largest LCD displays commercially available is 100 " on the diagonal [15]. As this

Permission to make digital or hard copies of all or part of this work for personal or classroom use is granted without fee provided that copies are not made or distributed for profit or commercial advantage and that copies bear this notice and the full citation on the first page. To copy otherwise, or republish, to post on servers or to redistribute to lists, requires prior specific permission and/or a fee.

CHI 2009, April 4-9, 2009, Boston, Massachusetts, USA.

Copyright 2009 ACM 978-1-60558-246-7/09/04...\$5.00. trend continues, it is highly likely that in the near future a user can easily turn entire walls into a high-resolution display surface. Today, we can easily achieve this large size, but not the high-resolution, by using an inexpensive projection display. Tiling multiple projectors increases the resolution, but at the cost of additional complexity in rendering and stitching the multiple images into a seamless whole. This is viable for use in research labs conducting studies such as the current one, but not for widespread use.

Most current computer users use just one or two standard monitors, which cover roughly $10 \%$ of their visual field [10]. When switching to a large display that can take upwards of $70 \%$ of the visual field, users may behave differently and indeed expect the system to provide very different interface support. To effectively utilize a large high-resolution display for daily work, we need to understand the aspects in which the increased size and resolution can benefit users, how a user will make use of the greater screen real estate, and how to refine interfaces to better suit these displays.

In this paper, we present a study that investigates users' behaviors when they switch from standard computing environments (i.e., single- or dual-monitor) to using a large high-resolution display (Figure 1) for a five day period. In particular, we looked at how users utilized and partitioned screen real estate, and managed windows in different display configurations. Based on the results, we formulate guidelines for large display interface design. Note that this study analyzes users' behaviors on a 16 'wide x 6'high, 6144 x 2034 resolution display with self-adjusted sitting distances. Additional controlled experiments are required to further clarify how physical size, number of pixels, and field of view of a display could affect users' behaviors.

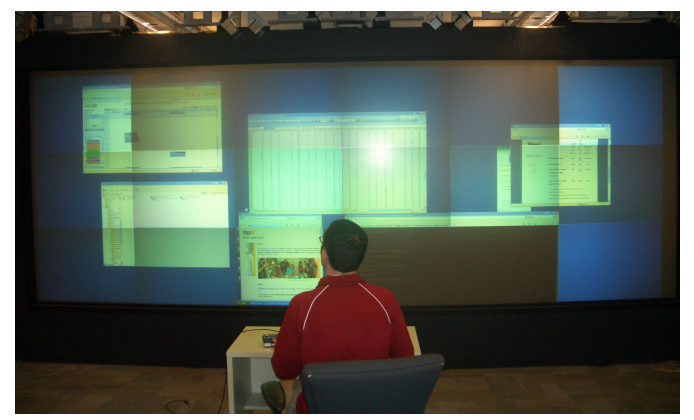

Figure 1. User working on a large high-resolution display in our diary study. 


\section{RELATED WORK}

Despite various large displays being widely used, very little work has been done to investigate how large display might be used in the context of daily desktop computing. Ball and North [1] observed the use of a large tiled display comprising nine 17" LCDs (a 3 X 3 title) for personal desktop computing. Their results show that a large display affords a number of advantages, such as improving tasks switching, and enhancing users' awareness for secondary tasks. Bishop and Welch [8] reported their initial experiences in using projected screens instead of conventional monitors in a personal office situation. They concluded that "It's so great and we never want to go back to conventional displays". Czerwinski et al. [9] conducted a controlled experiment to compare a tiled large display comprised of three 21 " LCDs with a standard monitor, showing that a large display can significantly improve user performance for complex, multi-application office work. Distinct from this prior work, our study compares a large display with both single- and dual-monitor use by analyzing users' daily activities and mouse and window event logs, revealing unique styles of partitioning the screen and managing windows on a large display.

Besides daily computing tasks, a large display might also be beneficial in other tasks: it outperforms a normal size screen in spatial and virtual path selection tasks $[24,25]$; it improves female users' 3D navigation performance by providing a wider field of view [23]; it can prompt physical navigation and reduce the need for virtual navigation, thus improving performance in navigation tasks [3]; it can also enhance user performance in visualization tasks $[2,26]$.

In addition to empirical studies, prototypes involving the use of a large display have been designed and implemented to assist users processing daily work. In the office of the future [19], a user can designate real surfaces in the office to be used as spatially immersive display surfaces. Dynamic changes in the surfaces could be interpreted for the purpose of tracking, interaction, and augmented reality applications. In Kimura [17], large projected displays are used in conjunction with a normal monitor to assist multitasking practices by showing montages comprised of images from current and past interaction on the desktop. Swaminathan and Sato [22] designed a prototype called Prairie which utilized six 29" displays to support a knowledge worker. The positive user experiences convinced the authors that it was simply a matter of time before large displays will be standard for home and office computers.

However, since most current interfaces are designed to suit a standard monitor, users encounter various usability problems when working on a large display, including: keeping track of the cursor [5], distal access to windows and icons $[7,16]$, and window management $[6,21]$. Some interaction techniques have been proposed to address these usability issues. [4, 5, 6, 7, 16, 20, 21]. As we further develop interfaces geared toward large displays, it is crucial that such efforts be guided by a deeper understanding of usage patterns and the usability problems faced.

Besides using a large high-resolution display, multiple monitors can also be used to expand screen real estate. Grudin [10] documents the usage patterns of dual-monitor users, revealing that generally a second monitor plays a supporting role and can improve work efficiency. Hutchings et al. [11] deployed a tool called VibeLog to a group of single and multiple monitor users, logging window management activities. Their results show the differences and similarities between two groups, enabling the formulation of dual-monitor interface design guidelines. Interaction techniques such as mudibo [13], QuickSpace [12], and window snipping [14] have been explored to facilitate dual-monitor use.

While there are similarities between using a multiple monitor approach to increasing screen real-estate and using a single high-resolution large display, there are also significant differences. First, the bezels of multiple monitors introduce a significant seam into the overall screen and consequently into the viewing experience, whereas a single large high-resolution display is seamless. Second, the physical sizes can be quite different, even if the number of pixels are similar. Hence, prior research on multiple monitor usage $[10,11]$ can at best serve as a very rough guide to single large high-resolution display usage; research explicitly focused on the latter is clearly required, and the present study takes one step in this direction.

\section{STUDY}

Our goal is to investigate how a person might use a large high-resolution display for daily work. In particular, we aim to compare large display use with traditional personal computing environments (i.e., single- or dual-monitors). Rather than conduct a controlled experiment to examine individual aspects in isolation, we carried out a diary study in a more realistic context, allowing us to explore usage in a broad range of computing activities over a five day period.

\section{Participants}

We recruited eight participants: four who used a singlemonitor, and four who used a dual monitor configuration for their daily computing. Two single-monitor users (S1, S2) usually worked on a $17^{\prime}$ ' LCD, and the other two (S3, S4) used a 21" LCD in their daily work. Two of the dual monitor users (D1, D2) worked on two 18" LCDs, one (D3) used a 21 ' in conjunction with a 18 ' LCD, and one (D4) used two 21" LCDs. S4 and D2 are female and the others are male. All used computers over 5 hours per day. We chose experienced computer users as we wanted to see how their interaction and visualization strategies might change when they move to a single large high-resolution display; in contrast, inexperienced users would not have a baseline of strategies that they would need to adapt when moving to our large display setup. 


\section{Apparatus}

We used a 16' wide x 6' high display (Figure 1), comprised of 18 projectors in a $3 \times 6$ tiling, mechanically to create a geometrically seamless image. We did only basic color and brightness calibration, hence there was some variation in brightness and color between each projector's part of the overall image. Each projector had a resolution of $1024 \mathrm{x}$ 768 pixels, for a total display resolution of 6144 x 2304 pixels. This display is significantly larger and higher resolution than those used in earlier large display studies [1, 8 , 9]. As there is no operating system specific to large displays, we ran standard Windows XP on a computer with several multi-headed graphics cards that could drive the multiple projectors concurrently. Various applications software was also installed to ensure that participants could perform their daily work.

\section{Design}

Participants were categorized into two groups according to their normal computing environments: single-monitor or dual-monitor users. Participants switched to exclusively use the large display as their daily computing environment for five consecutive days, with 5 hours per day. Thus, each group had two working conditions: their normal working environments (i.e., single or dual monitors) as well as the large display condition. The study ran over eight weeks period, with one participant using the display each week.

Participants were asked to perform their daily routine work on the large display. Throughout the study, the participant was the only person in the large display room, ensuring a personal office scenario and maintaining the user's privacy. Since the optimal position and distance of using the large display was unclear, participants could freely adjust the sitting position and distance in the study.

To understand how a user worked on the large display, we employed the following observation methods:

\section{Activity Log}

Each participant maintained a daily activity log when working on the large display, in which she wrote down the activities every half an hour. Figure 2 shows parts of a daily activity $\log$. The participant briefly described the activities occurring in the last half an hour, recorded the approximate distance to the display, and listed the advantages/disadvantages of undertaking these activities on the large display. Finally she ranked the large display as "better", "worse", or "equal", in comparison to performing these activities in her normal computing environment.

\section{Daily Interview}

To gain further insight of users' activities and examine the issues not covered by the daily activity log, a follow-up structured interview was carried out at the end of each day. The interview occurred right after the participant finished their daily work on the large display. The whole process usually took one hour, and was taped for later analysis. In the interview, participants were asked to elaborate on the events recorded on the activity log, describe screen real estate usage, and talk about the pros and cons of using a large display. Throughout we asked the users for more detail if the explanations given were unclear. However, in order not to bias the outcome, we paid special attention to ensure that the additional questions were non-suggestive and only clarifying in nature.

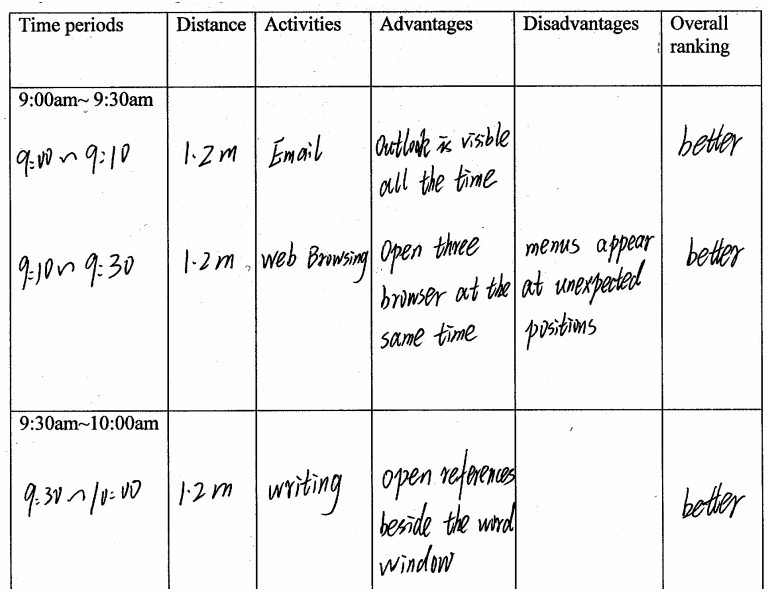

Figure 2. Example activity log

\section{Recording Windows and Mouse Events}

We ran an application called VibeLog [11] on the large display to record every window event that occurred and detailed information of each running window. It maintained two kinds of logs: events log, and windows log. The events $\log$ had an entry for every window management event that occurred. These activities included closing, activating, moving, resizing, minimizing, and maximizing a window. Each log entry had a timestamp and the title of the window operated. Complementarily, the windows log created a series of entries each minute, one entry for each open window on the system. The entry contained detailed information about the corresponding window, including the spatial coordinates, size, status of the window, and the window's z-order on desktop.

We also recorded every mouse event using an application written within our lab called MouseLog. It created an entry for every mouse activity, which contained the spatial coordinates of the mouse cursor, and event type (e.g., left button down/up, right button down/up).

Both VibeLog and MouseLog worked by programmatically hooking into the public window system events made available by Windows XP. They occupied little system resources and did not interfere with normal computer use.

These four recording means are complementary to each other. The daily activity log provides data about a participant's real-life activities in workplace, while the follow-up interview extends those results with more qualitative descriptions. However, since the activity $\log$ and interview both require that participants self-report their activities after the fact, they may fail to recall all activities 
of interest, or their beliefs about the study's purpose may bias their selection of events to report. Complementarily, the VibeLog and MouseLog objectively record user activity, which can not only corroborate the results drawn from the activity $\operatorname{logs}$ and interviews, but also reveal interesting activities that might be missed by the self-report.

Note that before a participant switched to using the large display, VibeLog and MouseLog were deployed in her normal computing environment for five working days to record windows and mouse events. This data served as baseline. When working on the large display, all four recording methods were employed to gather richer data. All participants reported that they were doing similar work during these two weeks (one week in the normal computing environment, and the other on the large display). We did not do the activity $\log$ or daily interview during the five days of standard computing environment usage as we felt it would impose undue demands on our participants who were already committing significant time to this study, while any new insights gained would likely be minimal since standard computing environments have already been well studied.

\section{Results}

Rather than report the results gleaned from each of our four logging methods separately, we instead present a more holistic analysis that extracts the most interesting findings from all our data taken as a whole.

\section{Overall Activities}

Table 1 shows the categories of activities and the time logged on those activities across all the participants on the large display. The categories that account for most of the hours are web browsing, word processing and reading papers, with each of them constituting more than $20 \%$ of the total hours logged. Moreover, all eight participants performed web browsing, word processing, reading papers, and emailing tasks. These four tasks take up nearly $80 \%$ of the hours logged.

\begin{tabular}{|l|r|r|c|}
\hline Activities & \multicolumn{1}{|c|}{ Hours } & Percentage & $\begin{array}{l}\text { \# of participants } \\
\text { reporting activity }\end{array}$ \\
\hline \hline Web Browsing & 54.5 & $25.9 \%$ & 8 \\
\hline Word Processing & 48.3 & $23.0 \%$ & 8 \\
\hline Reading Paper & 42.0 & $20.0 \%$ & 8 \\
\hline Emailing & 21.8 & $10.4 \%$ & 8 \\
\hline Programming & 12.4 & $5.9 \%$ & 5 \\
\hline $\begin{array}{l}\text { Data Analysis } \\
\text { (Excel) }\end{array}$ & 10.5 & $5.0 \%$ & 3 \\
\hline $\begin{array}{l}\text { Preparing } \\
\text { presentation slides }\end{array}$ & 6.5 & $3.1 \%$ & 3 \\
\hline $\begin{array}{l}\text { Chatting (MSN or } \\
\text { GTalk) }\end{array}$ & 5.0 & $2.4 \%$ & 6 \\
\hline 3D modeling & 5.0 & $2.4 \%$ & 1 \\
\hline Graphic Drawing & 4.5 & $2.1 \%$ & 2 \\
\hline Total & 210.5 & $100 \%$ & \\
\hline
\end{tabular}

Table 1 Hours logged per major activity on the large display

\section{Subjective Opinions}

Figure 3 shows each participant's subjective opinion comparing the large display with their normal computing environments. Perhaps somewhat surprisingly, all participants overwhelmingly preferred the large display: for each participant, "Better" and "Equal" constitute more than $90 \%$ of the rankings and more than $50 \%$ of the rankings are "Better". All participants reported hoping to obtain more screen space for their computers. One dual-monitor user (D1) commented:

"When I was working on a 15 inch monitor, I thought a 19 " LCD would be much better. When I switched to a 19", one, I found it was still too small. Now I am using two 18", monitors, but I still hope to get more screen space"

In particular, single-monitor user S4 always ranked the large display "Better" or "Equal" Her activities on the large display included web browsing, word processing, emailing, on-line chatting, and $3 D$ modeling. She expressed a strong preference for working on the large display, commenting:

"The larger one is pretty better. It [the large display] offered me much flexibility of doing daily work. I can choose the optimal amount of screen space according to the task at hand."

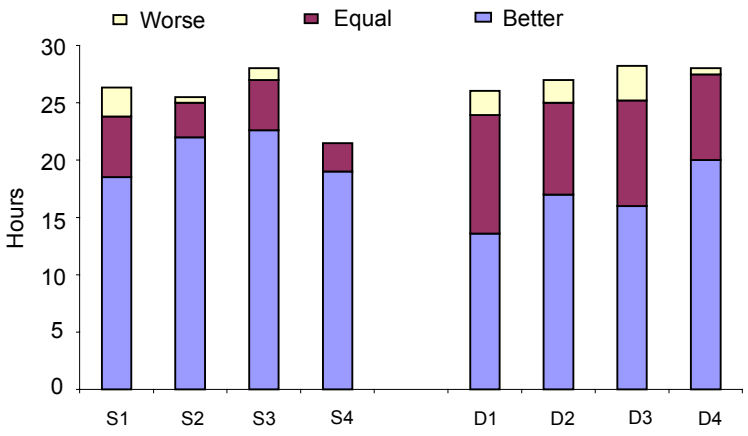

Figure 3. Participants' subjective opinions of the large display compared to the traditional single- or dual-monitor configuration. (S1, S2, S3, and S4 represent four singlemonitor participants, while D1, D2, D3, D4 are four dualmonitor users)

Figure 3 also demonstrates that single-monitor users have a stronger preference for using the large display than dualmonitor users. Single-monitor participants rated the large display "Better" for $81 \%$ of hours while dual-monitor participants did so only for $61 \%$ of the working time. The relatively smaller screen space on a single monitor may make single-monitor users feel like they are benefiting more from the large display than dual-monitor users.

Figure 4 breaks down the rankings by task. Both single- and dual-monitor users unanimously prefer the large display to their normal computing environments across all the tasks: for each task, "Better" and "Equal" constitute more than $80 \%$ of the rankings. In particular, the large display is always rated "Better" or "Equal" for programming, data analysis (excel), and 3D modeling. 


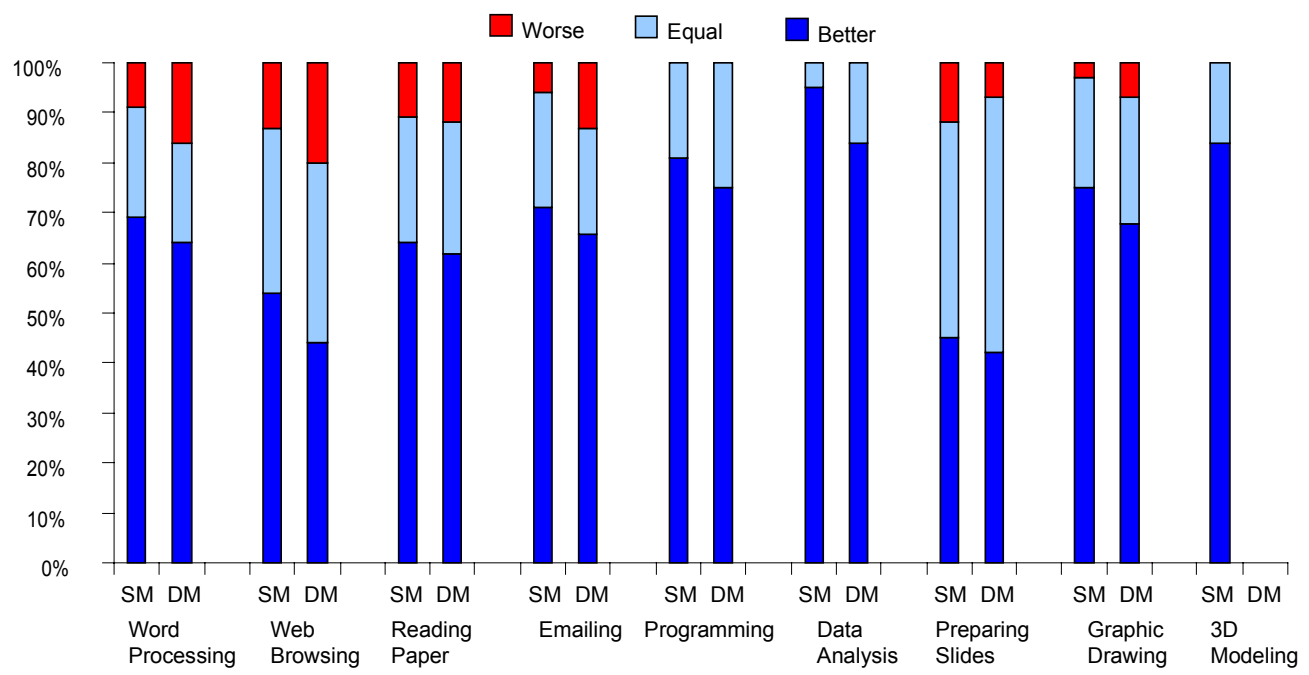

Figure 4. Participants' subjective opinions of the large display compared to the traditional single- or dual-monitor configuration, distributed by task. (SM and DM represent the four single-monitor and four dual-monitor participants, respectively).

Three single-monitor (S1, S2 and S3) and two dual-monitor users (D1 and D3) performed programming tasks on the large display. All reported preferring the large display, because it allowed them to simultaneously view multiple windows, which they felt improved their work flow. For example, D1 coded in JAVA on the large display. He kept a Java NetBeans window, an on-line help document, an electrical dictionary, and a Google search webpage visible concurrently. The Java NetBean was the primary coding window, the on-line help document and the dictionary helped him to check unfamiliar Java functions, and the search page was used to search other help information from the internet. Keeping all these four windows visible eased the access to any of them. In contrast, in his normal dualmonitor setup, he can keep just two of these applications visible, thus requiring frequently interleaving actions.

Spreadsheet data analysis receives the highest "Better" ranking (91\%). Two dual-monitor (D2 and D4) and one single monitor user (S2) analyzed data using Excel on the large display. All of them reported benefiting significantly from the larger display, because of the huge number of spreadsheet cells that could be kept visible simultaneously.

One single monitor user (S4) used Autodesk's Maya to build 3D models on the large display. She rated the large display "Better" for more than $80 \%$ of her time. The large display can concurrently visualize the several sub-windows she had to use concurrently while working in Maya, thus significantly improving the work flow.

The large display received the highest "Worse" ranking (9\%) for web browsing tasks. Participants reported that rendering a large image was slow and there were some usability problems with the web browser (Internet Explorer) as some interface elements did not scale correctly to the large display. For example, when a web browser window was enlarged, the content inside was not enlarged as expected. Also, activated menus may appear at unexpected positions.
Focal vs. Peripheral Regions

All the dual-monitor participants reported mentally partitioning the screen real estate into focal and peripheral regions in both the dual-monitor and large-display, while single-monitor participants did so only on the large display. Generally, the focal region is used for primary tasks writing code, word processing, or drawing a graphic image - to which most interaction activities are devoted over time, while the peripheral region is used for applications that are secondary to the primary tasks, such as email clients, instant messaging clients, and personal "to do" lists.

While the dual-monitor participants partitioned screen space in both the dual-monitor and large-display conditions, they did so differently in these two circumstances.

The distribution of mouse events shows differences in location of both focal and peripheral regions (Figure 5). On dual monitors, participants performed more activities in one monitor than in the other: $71 \%$ of mouse events occurred within one monitor, and $29 \%$ occurred in the other. According to dual-monitor participants' reports, one monitor was usually used as the focal region, and the other as the peripheral. Due to monitors' physical bezels, spanning a window across two monitors suffers from visual discontinuities, so the primary activities were usually restricted to one monitor. However, when dual-monitor participants worked on the large display, $81 \%$ of the mouse events occurred in the center region of the large display, and $19 \%$ occurred in the remaining "inverted-U" shaped area (Figure 5). The center region was used as the focal region, while the rest of the display was used as the peripheral region. This distribution might be explained by users' sitting positions. As all the participants sat in front of the horizontal centre of the large display, the center part is the closest region to the users, making it convenient to view and interact with. Consequently, users are more likely to position focus-required work in this part, and peripheral applications requiring less attention slightly further away. 

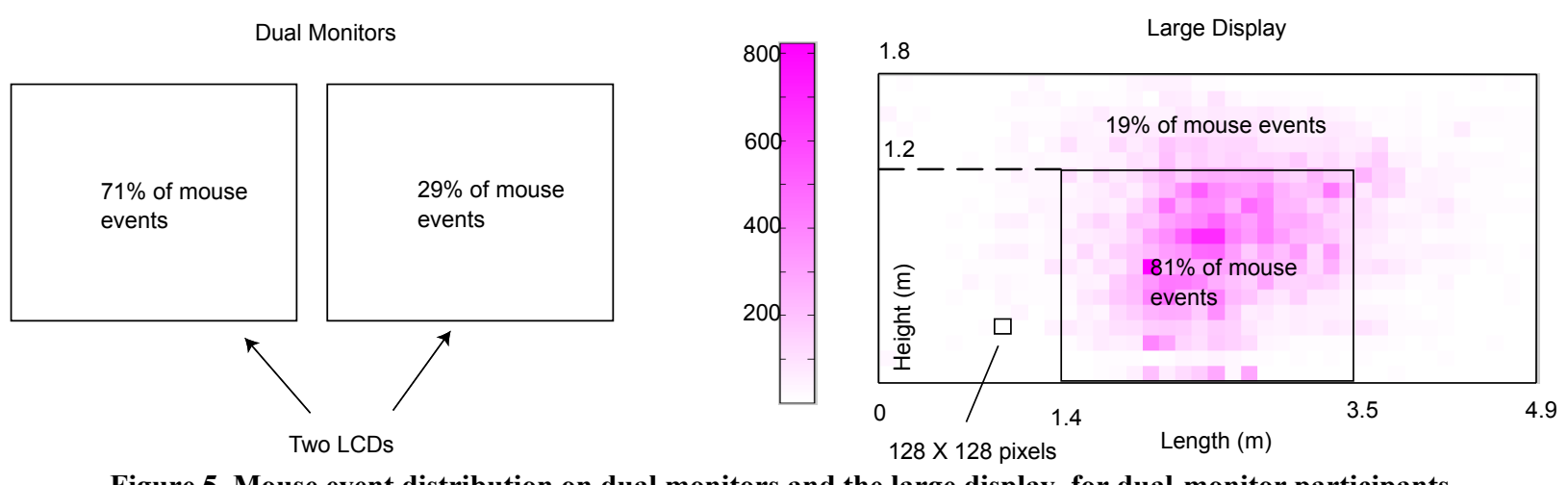

Figure 5. Mouse event distribution on dual monitors and the large display, for dual-monitor participants

\section{Arrangement of Application Windows}

Aside from differences in location of focal and peripheral regions, participants managed, interacted with, and displayed applications on the large display in distinct ways than on dual monitors

One major distinction is related to how users arranged their application windows. Due to the relatively limited and divided screen space on the dual monitors, participants usually invested little effort in arranging application windows in this condition. One dual-monitor participant (D2) reported:

"I just throw all the non-primary applications to the secondary monitor, and do not care much about the layout. If I need to use one of them, I maximize it by a simple click."

However, on a large display, participants tend to expend more effort to optimize the layout of application windows to improve workflow. One participant reported that at the beginning of each day, he spent nearly 5 minutes arranging the applications. He commented:

"It takes a certain amount of time at the beginning of each day, but worthwhile, because this optimal layout help me greatly afterwards".

In particular, post-interviews revealed that there are two common strategies for arranging windows. The first is to position the applications requiring interaction activities, such as MS Word and instant messaging clients, close to the center of the screen, while the applications only passively displaying information, such as a weather forecast window or a calendar, on the side or corner of the screen. Figure 6 shows a layout using this strategy captured during one of the participant's usage sessions. This strategy aims to facilitate the interaction with the applications beside the focal region.

The second strategy is to arrange windows according to their relevance to the primary task. The more relevant, the closer the application is to the primary task window. For example, when drawing a car, the canvas window was positioned in the center, and surrounded by applications displaying reference materials. Other applications less relevant to the drawing process were placed further away.
Interacting with Applications in the Peripheral Region

Another difference is how users interact with peripheral applications. On dual monitors, users just slightly turned their heads and bodies to work on applications on the secondary monitor, such as email and instant messaging clients. These applications were dragged into the primary monitor only when they would occupy interaction focus for a long period of time. This behavior occurred because two monitors were usually placed close to each other, requiring only a slight head or body movement to shift attention.

In contrast, when interacting with peripheral applications on a large display, users often dragged them from the peripheral into the focal region, and seldom turned their heads and bodies. This action likely resulted from these peripheral applications usually residing on the side or corner of the large display, thus requiring plenty of head and body movements to shift attention. Also, concentrating on distant applications is difficult, which discourages working with them in the peripheral region.

Take replying to emails for example. On dual monitors, the email client usually resided on the secondary monitor. A user (D3) just slightly rotated his head and body to reply to the email whenever a new message arrived. However, when working on the large display, he frequently switched the email client back and forth between the focal and peripheral regions: dragging the email client into the focal region to reply, and then sending the application back to the previous location afterwards. He commented:

"Although dragging the application back and forth between side and center region is time consuming, it is still more comfortable than rotating my head and body to interact with it".

\section{Visualizing Applications in the Peripheral Region}

On a large display, since the peripheral region is distant from seating positions and viewed from more acute angles, pictures and text in this region are usually magnified to ensure clarity and facilitate viewing. As seen in a screen shot (Figure 6), the picture within the weather forecast window is magnified by $200 \%$, and the font size for the "to do" list is enlarged to 25 from the usual 10 on a normal desktop screen. A similar enlargement of peripheral content did not occur in the dual-monitor condition. 


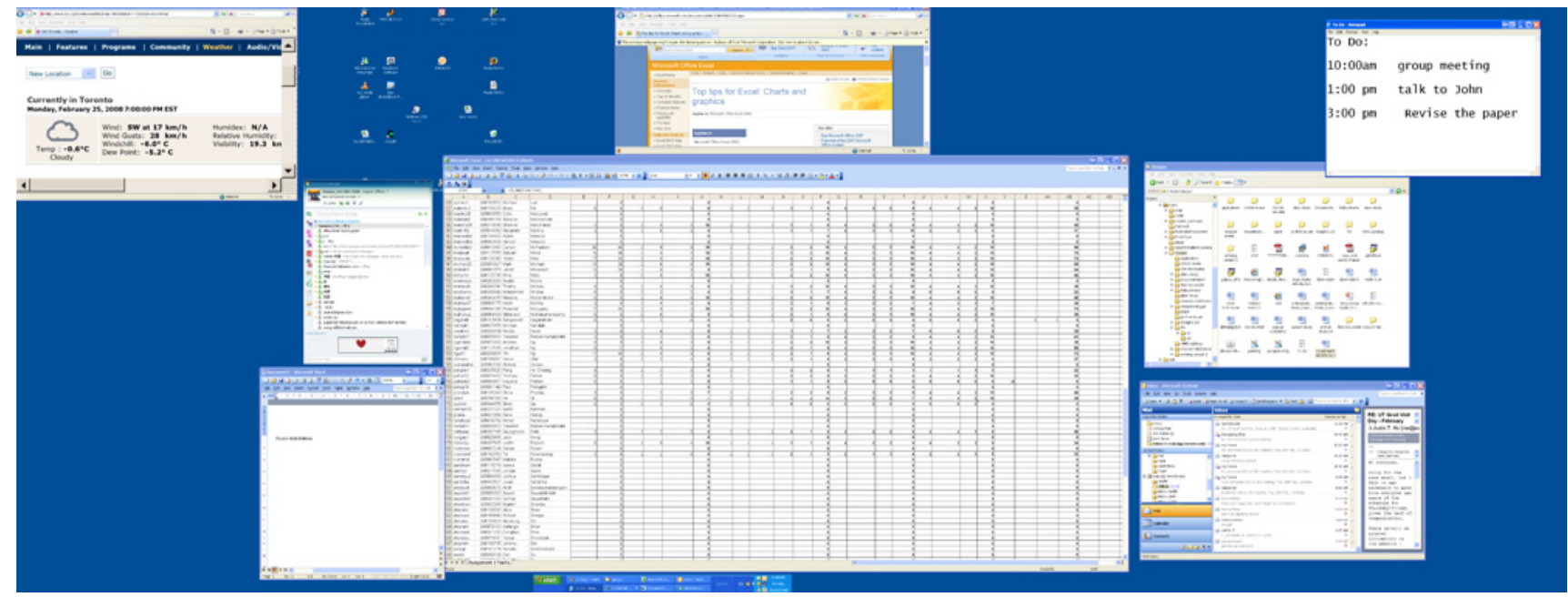

Figure 6. A screen capture of the large display from one participant's session, illustrating how the screen real estate is implicitly partitioned into focal and peripheral regions.
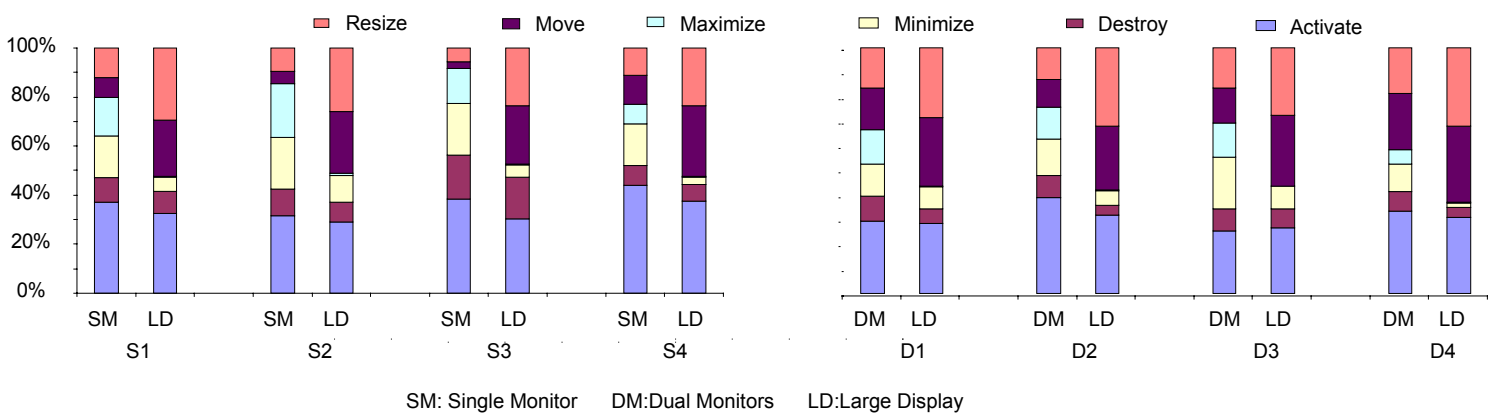

Figure 7. Distribution of window management operations per participant. (S1, S2, S3, and S4 represent four single-monitor participants, while D1, D2, D3 and D4 are four dual-monitor participants).

\section{Window Management Operations}

On standard desktops, users typically partition screen real estate via manipulating windows. To gain a deeper insight into screen space usage, we compared windows management operations across the different conditions.

Figure 7 shows the distribution of different window management operations for each person. One common characteristic across all the participants is that moving and resizing operations constitute much higher percentages of total operations on the large display than in their normal computing environments. For single-monitor users, the mean percentage of moving plus resizing operations is $51.5 \%$ (std. dev. $=2.4 \%$ ), on the large display and $16.7 \%$ (std. dev. $=6.1 \%$ ) on the single monitor; for dual-monitor users, it is $58.5 \%$ (std. dev $=2.4 \%$ ) on the large display and $29.5 \%$ (std. dev. $=9.7 \%$ ) on the dual monitors. Whenever a new application was opened, the following operation was to optimize its position and size. Moreover, frequently switching applications back and forth between the peripheral and focal regions also causes more moving operations on the large display.
Another interesting finding is that dual-monitor participants performed more window moving and resizing operations than single-monitor participants in their normal computing environments. However, when using the large display, both single- and dual-monitor participants performed similar percentages of moving and resizing operations, which were much higher than those in their normal environments.

In contrast to moving and resizing, maximizing and minimizing operations constitute a much lower percentage of actions on the large display. Only one (S2) out of eight participants ever maximized windows on the large display. S2 did so to show a Google map. Other participants reported never spanning a window across the entire large display surface. When they needed to visualize windows containing rich information, $60 \sim 70 \%$ of the entire display surface was typically sufficient. This is a particularly interesting finding in that it indicates an upper bound on window sizes that users are comfortable working with. Minimizing operations were often performed in single- or dual-monitor conditions to save screen space, but it was rarely used on a large display due to the ample available space. 


\section{Seating Location Relative to the Display}

As the optimal seating location when working with a large display is unclear, participants were allowed to freely adjust their seating during the study. Wheels were mounted on the table and chair to enable easy mobility. All of the participants reported sitting in front of the horizontal centre of the display, because they felt that their visual fields covered most of the screen space from this position. Figure 8 shows the durations of all the sitting distances from the display which were self-reported by filling the activity log. As shown, the distances range from $0.9 \mathrm{~m}$ to $3.0 \mathrm{~m}$, with more than $90 \%$ of data between $1.5 \mathrm{~m}$ to $3.0 \mathrm{~m}$. Most relevant is that participants sat within $2.0 \mathrm{~m}$ to $2.5 \mathrm{~m}$ of the display nearly $50 \%$ of the time.

When participants worked in their normal computing environments, the distances to the screen ranged from $0.75 \mathrm{~m}$ to $0.85 \mathrm{~m}$. All participants reported sitting slightly further when switching to the large display because they wanted to view more of the screen at once. One single- (S2) and one dual-monitor participant (D3) reported that sitting too close (less than $1.0 \mathrm{~m}$ ) to the large display made them feel like sitting facing upclose to a wall, which was very uncomfortable. However, sitting too far away can also make viewing details on the display difficult. It seems that a distance of $1.5 \mathrm{~m}$ to $2.5 \mathrm{~m}$ is the optimal range for most of the participants, a distance at which they can clearly perceive the content on the large display and their visual field covered sufficient screen real estate.

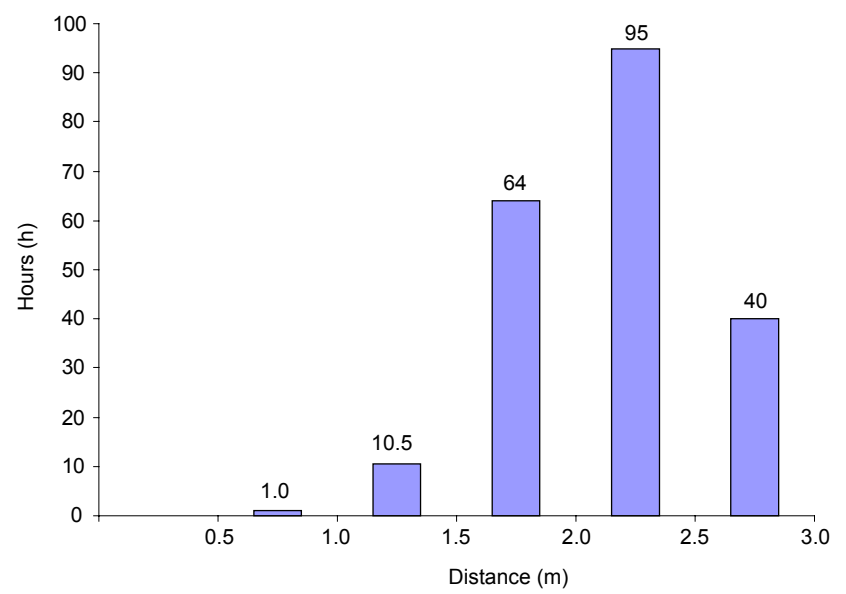

Figure 8. Time spent at different distances to the large display

\section{DISCUSSION}

\section{Benefits of Large Displays}

As shown in Figure 3, despite the fact that the Windows XP operating system is designed for a normal desktop screen, participants still overwhelmingly preferred a large display to their normal computing environment (i.e., single- or dual- monitors). Our results indicate that a large display can provide several key benefits:

\section{Multi-window Tasks}

A large display benefits multi-application tasks by enabling the simultaneous display of multiple windows. As most of the relevant windows are visible, window interleaving operations that frequently occur in single- or dual-monitors situations could be reduced. Previous research [9] has shown that a large display (albeit one that was much smaller and of lower resolution than the display in our study) outperforms a small display for complex, multiapplication office work. Complementarily, our study shows that users intentionally optimize window layout to facilitate multiple-window collaboration to improve their workflow.

\section{Rich Information Tasks}

Another obvious benefit is related to applications containing rich digital information, such as spreadsheets and digital maps. A large display can visualize more information than single- or dual-monitors, thus reducing the navigation overhead. One participant (D2) expressed strong preference for analyzing data in Excel files on the large display, because it can show all the columns. In his usual dual-monitor setup, he had to frequently scroll left and right, up and down in the application window because only partial information was visible.

\section{Awareness of Peripheral Applications}

Being aware of peripheral applications could improve work productivity [18]. A large display can fully take advantage of user's peripheral vision by showing numerous peripheral applications. For example, as shown in Figure 6, the user opened a weather report, "to-do" list, email client, and instant messaging client in the peripheral region. He could maintain awareness of these four peripheral applications while working on the primary task. In contrast, in his usual single-monitor environment, due to the limited screen real estate, only the major application window was fully visible. Peripheral applications were usually obscured.

\section{Immersive Experience.}

Tan et al.,'s work [24] shows that a large display outperforms a normal monitor in 3D navigation tasks because of the immersive experience generated by the large display. Although users' personal desktop work is not typically comprised of 3D navigations tasks, participants reported that a large display engaged them more in the daily work than single- or dual-monitors. When sitting in front of the large display, they felt "surrounded" by the task. This feeling helped them to focus attention on the task, especially when they were performing attentive work such as proof-reading, or coding. Additionally, the large display might provide some ergonomic benefits. In the single monitor condition, the user's head and eyes are restricted to the limited screen space, which might easily cause fatigue if working for a long period of time. However, users felt more relaxed using a large display because they can freely adjust hand and body positions while maintaining a good view of the display. 


\section{Design Implications}

Our study reveals that, compared to single- or dualmonitors, users operate differently on a large display in partitioning screen real estate and managing windows. To better support the types of user operations that we observed in our study, user interfaces and window management systems need to be refined; our study's results can be used for formulate recommendations for such refinement. Note that although some work $[1,20]$ has been done to guide large display interface design, we develop our design recommendations from the standpoints of screen space partitioning and window management operations, which have not been previously articulated in the literature.

\section{Peripheral vs. Regular Modes of an Application}

Compared to applications in the focal region, applications in the peripheral region serve a different function providing peripheral awareness of some information that requires monitoring, and have specific visualization and interaction requirements on a large display. To accommodate this diversity, we suggest that each application might operate in two modes: regular and peripheral. The former works for applications in the focal region, and the latter for those in the peripheral region. Distinct from the regular mode, applications in peripheral mode would have following characteristics:

High Legibility. First, applications should always be legibly displayed. Since the peripheral region of a large display is far from a user's sitting position, increasing the size of the imager within peripheral windows would ease the viewing process. Moreover, high legibility ensures glancibility - the ability to absorb information with a quick look. A simple approach for achieving high legibility is to automatically magnify pictures and text inside an application when it switches to the peripheral mode

Quick switching and moving. Second, large display users desire a method to quickly switch applications back and forth between the peripheral and focal regions. According to our study results, this application switching activity occurs frequently on a large display. However, moving an application on a large display using traditional techniques is time-consuming and can cause finger fatigue, because it requires dragging the mouse a long distance as well as holding the mouse button down for a long period of time. Easing this switching process could significantly improve window management efficiency. One simple approach might be adding a switching button on each application window. Clicking it will bring the application from the peripheral region into the focal region and clicking again will send the application back.

Simplified Layout. To facilitate peripheral information absorption, a window's layout should be simplified to emphasize the necessary information and avoid distracting decorations. A first step might be to automatically hide the menus and icons of applications in peripheral mode, a strategy that is simple but very effective especially when a window is shrunk. In our study, we observed that the menus and icons on the top of an application are distracting when the application window is condensed. For example, as shown in the top left corner of Figure 6, menus and icons take up almost half of the weather forecast window's space. Since this application just passively displays weather information, these menus and icons are almost functionally useless yet distracting. Hiding menus and icons not only saves screen space but also emphasizes the weather information inside. In case the user wants to use these menus or icons, moving the cursor over the top of the application for a few seconds would bring them up. Hutchings et al. [14] describe a windows snipping technique to simply show the required part of a window. This technique could be useful in a large display environment.

Additional window operations. In addition to the different screen space partition behaviours, our study reveals windows management differences between the large display and single- or dual-monitor conditions. On a large display, users are more likely to resize and move windows, and less likely to minimize and maximize them. We suggest that a large display window management system should concentrate on facilitating the most frequently executed operations (i.e., resizing and moving windows), and deemphasize less used operations (i.e., minimizing and maximizing windows).

Traditionally, resizing a window is performed by dragging a window's corner. Many participants encountered inconveniences when resizing a window on a large display: accurately selecting the corner of an application in the peripheral region was difficult, and holding the mouse button and dragging a long distance was tended to be prone to fatigue. A lighter-weight resizing approach might significantly benefit users. For example, a user could move the cursor to an icon on the corner of a window and turn the scroll wheel down/up to resize it.

Since maximizing and minimizing buttons are rarely used on a large display, we could replace these two buttons with other more frequently used functions. For example, replacing the maximize button with an optimal size button, and the minimize button with a quick switching function.

\section{CONCLUSION AND FUTURE WORK}

We have presented a week-long study comparing usage of a large display to single- and dual-monitor configurations in a daily work environment. Results indicate that users unanimously prefer using a large display. In particular, a large display could benefit multi-window tasks and richinformation applications, enhance users' awareness of peripheral applications, and offer immersive working experiences.

With the huge amount of screen real estate, users tend to utilize the center part as the focal region, and the remaining space as the peripheral region. The results also reveal that users on a large display perform more window moving and 
resizing, but less minimizing and maximizing operations as compared to a single- or dual-monitor. Detailed analysis of these findings provides implications for designing appropriate interfaces and window management systems for future large displays.

Our approach of enhancing the self-report data (i.e., activity logs and interviews) with objectively recorded mouse and window event logs provides cross-validation for many of our findings. However, since every participant switched from the normal computing environment to a large display, this change of working environments might have inadvertently affected the participant's behaviours. In addition, the usage patterns revealed by our one-week long study period may need to be further verified by a longer term study, and specific implications drawn from this study might benefit from further refinement and verification. We hope to explore these in our future work.

\section{ACKNOWLEDGEMENTS}

We thank Microsoft Research for offering the VibeLog software.

\section{REFERENCES}

1. Ball, R. and North, C., (2005). An analysis of user behavior on high-resolution tiled displays. IFIP International Conference on Human-Computer Interaction. p. 350-364.

2. Ball, R. and North, C. (2007). Effects of tiled highresolution display on basic visualization and navigation. ACM CHI Extended Abstracts. p.1196-1199.

3. Ball, R., North, C. and Bowman, D.A. (2007). Move to improve: promoting physical navigation to increase user performance with large displays. ACM CHI. p.191-200.

4. Baudisch, P., Cutrell, E., Robbins, D., Czerwinski, M., Tandler, P., Bederson, B. and Zierlinger, A., (2003). Drag-and-pop and drag-and-pick: techniques for accessing remote screen content on touch- and pen-operated systems. Interact. p.57-64.

5. Baudisch, P., Cutrell, E. and Robertson, G., (2003). Highdensity cursor: A visualization technique that helps users keep track of fast-moving mouse cursors. Interact. p. 236243.

6. Bezerianos, A. and Balakrishnan, R. (2005). View and space management on large displays. IEEE Computer Graphics and Applications, 25(4). p. 34-43.

7. Bezerianos, A. and Balakrishnan, R. (2005). The vacuum: facilitating the manipulation of distant objects. ACM CHI. p. 361-370.

8. Bishop, G. and Welch, G. (2000). Working in the office of "real soon now". IEEE Computer Graphics and Applications, $20(4)$ ). p.76-78.

9. Czerwinski, M., Smith, G., Regan, T., Meyers, B., Robertson, G. and Starkweather, G., (2003). Toward characterizing the productivity benefits of very large displays. Interact. p. 9-16.
10. Grudin, J., (2001). Partitioning digital worlds: focal and peripheral awareness in multiple monitor use. $A C M C H I$, p. 458-465.

11. Hutchings, D., Smith, G., Meyers, B., Czerwinski, M. and Robertson, G. (2004). Display space usage and window management operation comparisons between single monitor and multiple monitor users. Advanced Visual Interfaces. p. 32-39.

12. Hutchings, D. and Stasko, J. (2002). QuickSpace: New operations for the desktop metaphor. ACM CHI Extended Abstracts. p.802-803.

13. Hutchings, D. and Stasko, J. (2005). mudibo: multiple dialog boxes for multiple monitors. ACM CHI Extended Abstracts. p.1471-1474.

14. Hutchings, D. and Stasko, J. (2007). Quantifying the performance effect of window snipping in multiplemonitor environments. Interact. p. 461-474.

15. LG LCDs, http://www.lge.com

16. Khan, A., Fitzmaurice, G., Almeida, D., Burtnyk, N. and Kurtenbach, G. (2004). A remote control interface for large displays. ACM UIST. p. 127-136.

17. MacIntyre, B., Mynatt, E., Voida, S., Hansen, K., Tullio, J. and Corso, G. (2001). Support for multitasking and background awareness using interactive peripheral displays ACM UIST. p. 41-50.

18. Matthews, T., Dey, A., Mankoff, J., Carter, S., and Rattenbury, T. (2004). A toolkit for managing user attention in peripheral displays. ACM UIST. p. 247-256.

19. Raskar, R., Welch, G., Cutts, M., Lake, A., Stesin, L. and Fuchs, H. (1998) The office of the future: a unified approach to image-based modeling and spatially immersive. ACM SIGGRAPH. p. 179-188.

20. Robertson, G., Czerwinski, M., Baudisch, P., Meyers, B., Robbins, D., Smith, G. and Tan, D. (2005). The largedisplay user experience. IEEE Computer Graphics and Applications, 25 (4). p. 44-51.

21. Robertson, G., Horvitz, E., Czerwinski, M., Baudisch, P., Hutchings, D., Meyers, B., Robbins, D. and Smith, G. (2004). Scalable fabric: flexible task management. Advanced Visual Interfaces. p. 85-89.

22. Swaminathan, K. and Sato, S. (1997). Interaction design for large displays. Interactions, 4(1). p. 15-24.

23. Tan, D., Czerwinski, M. and Robertson, G. (2003). Women go with the (optical) flow. ACM CHI. p. 209-215.

24. Tan, D., Gergle, D., Scupelli, P. and Pausch, R. (2003). With similar visual angles, larger displays improve spatial performance ACM CHI. p. 217-224.

25. Tan, D., Gergle, D., Scupelli, P.G. and Pausch, R. (2004). Physically large displays improve path integration in $3 \mathrm{D}$ virtual navigation. ACM CHI. p.439-446.

26. Yost, B., Haciahmetoglu, Y. and North, C. (2007). Beyond visual acuity: the perceptual scalability of information visualizations for large displays. $A C M C H I$. p.101-111. 